Massachusetts Department of Mental Health Barbara A. Leadholm, M.S., M.B.A., Commissioner

\title{
Psychoactive Medication for Children and Adolescents: Orientation for Parents, Guardians, and Others
}

The Children and Adolescents'

Psychoactive Medication Workgroup

Division of Child and Adolescent Services Office of Clinical and Professional Services

February 2002

Revised July 2003

Revised J anuary 2005

Revised September 2007 


\section{PREFACE TO 2005 REVISION}

This revision reflects new scientific data, policy developments, and feedback from users of the first version. Appreciation is expressed to Manuela da Costa, of DSS, and Dr Virginia Merritt.

Gordon Harper, MD

Medical Director

Child \& Adolescent Services

\section{PREFACE TO THE 2007 REVISI ON}

The revision has been updated to reflect new information about treatments and current controversies. Contributions from Mary Ellen Foti, MD, and Sean Palfrey, MD, are particularly appreciated.

Gordon Harper, MD

Medical Director

Child \& Adolescent Services 


\section{PREFACE}

This booklet presents principles for the use of psychoactive medication in children and adolescents. It offers information about medication treatment. It does not recommend specific medications or doses. The booklet was developed by a group convened by the Division of Child and Adolescent Services of the Massachusetts Department of Mental Health. The group included representatives of the Department of Social Services (DSS), the Division of Medical Assistance, the J uvenile Court Clinic, the Massachusetts Behavioral Health Partnership, the New England Council of Child Psychiatry, the Parent Professional Advocacy League, and representative hospitals and health care organizations. Participants are listed in Appendix VII, Members of Working Group.

The Working Group expresses appreciation for review of the document and helpful suggestions from other colleagues, notably Drs. Joseph Biederman, Ronald Steingard, Ed Wang, and Linda Zamvil, and Bernadette Drum, BSN, J D.

Gordon Harper, MD

Chairman

Children and Adolescents' Psychoactive Medication Workgroup 


\section{TABLE OF CONTENTS}

\section{I NTRODUCTION}

A. Childhood Disorders: How common?

B. Psychoactive Medication for Children and Adolescents: Recent changes

C. Psychoactive Medication for Children and Adolescents: How do we know what to recommend?

D. The FDA, "approved" and "off-label" uses, black box warnings, and clinical judgment

E. Personal Representatives: Their responsibilities

F. New Knowledge, New Ways to Help Children and Adolescents

G. Promoting Access

H. This Booklet

\section{CHI LDHOOD AND ADOLESCENT MENTAL HEALTH - AN OVERVIEW}
A. How children grow: Differences in development
B. When do "differences" amount to "disorders?"
C. What can medication help with?

\section{PRI NCI PLES OF PRACTI CE}

A. Assessment

1. Comprehensive assessment: What does that mean? 
2. Family, Peers and School, Pediatric Liaison, Psychological Testing

3. Interviews, Rating Scales: Using both

4. Providing more than a DSM diagnosis

5. Cultural assessment

B. Planning and Contracting for Treatment

1. Articulating Problems, Target Symptoms, and Goals

2. Selecting Medications

3. Planning Treatment and Monitoring: Monitoring, Anticipating Problems, Seeking Consultation

4. Informed Consent: Who, What, How, When, Where, To Whom?

C. Integration of Treatment

D. Communication and Record Keeping

E. Continuity of Care

\section{MEDI CATI ONS USED TODAY}
A. Stimulants
B. Antidepressants
C. Mood Stabilizers
D. Antipsychotics
E. Other Medications

\section{AREAS OF CONTROVERSY}
A. Antidepressants in Children and Adolescents
B. Attention Deficit-Hyperactivity Disorder
C. Bipolar Disorder
D. Use of Antipsychotics (also called Neuroleptics)
E. Post-Traumatic States 
F. Children and Adolescents with Developmental Disabilities

G. Extreme Behavioral Loss of Control (Aggressive and Self-Injurious)

H. Involuntary Treatment

I. Adverse Side Effects

\section{CLI ENT-PARENT EDUCATION}

\section{MEMBERS OF WORKI NG GROUP}




\section{I NTRODUCTION}

\section{A. Childhood Disorders: How common?}

Eleven percent of children and adolescents in the United States ages 9 to 17 are estimated to have a diagnosable mental or addictive disorder with significant functional impairment (Surgeon General's Report on Mental Health, 2000). Consistent with this estimate, 4 million youths may suffer from a major mental illness and have significant impairments at home, at school, and with peers. Those with impaired development are at greater risk. Abuse or neglect, suffered by three million children and adolescents each year in the United States, is a major risk factor for later mental disorder.

\section{B. Psychoactive Medication for Children and Adolescents: Recent changes}

Children and adolescents with serious emotional disturbance are receiving psychoactive medication more often than in the past.

Several factors have contributed to this increase. One factor is scientific progress; another is changing attitudes towards children and adolescents' mental health, such as:

- advances in our understanding of how the brain works. We now recognize that the brain functions differently in children and adolescents with mental disorders;

- evidence that many children with symptoms grow up to have disorders as adults;

- greater recognition of child and adolescent mental health needs and increasing readiness to define those needs in terms of brain functioning; and

- the availability of a wider range of psychoactive medications.

In addition, child and adolescent mental health services have changed:

- Children and adolescents with serious emotional disturbance are no longer treated only in hospitals, but often in foster homes and community residences, facilities that have not historically treated children and adolescents with serious behavioral symptoms. Children and adolescents are hospitalized for shorter lengths of stay, resulting in pressure to use interventions that are likely to have a rapid effect, such as medication.

\section{Psychoactive Medication for Children and Adolescents: How do we know what to recommend?}


Medication practice in children and adolescents, like other areas of medical practice, is based on clinical experience and controlled clinical trials. "Evidencebased medicine" includes both kinds of evidence.

While controlled clinical trials in children and adolescents are becoming more common, most medication practice in children-in general medicine as well as in mental health-is based on clinical experience and individual clinicians' judgment. This is because drug development for children and controlled clinical trials to evaluate those drugs are much more difficult to do than for adults. Therefore, most pharmacological research is done with adults.

\section{The FDA, "approved" and "off-label" uses, black box warnings, and clinical judgment}

The Food and Drug Administration (FDA) relies on information from controlled clinical trials to approve new medications. Because there are very few controlled clinical trials with children, the FDA has not approved the vast majority of medications for use with children. When medications are used for indications or populations (such as children and adolescents) that the FDA has not approved, such use is called "off-label."

In addition to approving medications, the FDA sometimes requires that warnings (so-called "black box warnings") be printed along with medication information that is included when a prescription is filled. (See sections IV.B. and V.A., for discussion of the "black box warning" regarding antidepressant medications.)

Clinicians prescribing for children and adolescents must exercise caution, considering the physical and emotional development of the child, any neurological or developmental deficits, possible interactions with other medications, and the risk that children or adolescents may try other drugs or alcohol.

Children's and adolescents' responses to medication are difficult to predict. A child may not respond to a medicine that has been helpful to others, or may require a higher or lower dose than usual. Another child may have side effects at a dose that most can tolerate well. Genetic differences may account for much of this variability.

\section{E. Personal Representatives: Their responsibilities}


Whenever a medication is recommended for a child or adolescent, those with responsibility for them, called Personal Representatives, ${ }^{1}$ should raise questions like these:

- How do I assess medication recommendations made by a clinician?

- With limited data about possible benefits and risks, particularly in growing children and adolescents, on what basis do clinicians make recommendations?

- How should I decide to authorize treatment?

- How can clinicians inform me of my choices?

- How can I get the best information?

This booklet responds to these questions. In doing so, we hope to contribute to one of the goals in the Surgeon General's Action Plan for Children's Mental Health: to "make available information on effective prevention and treatment interventions..."

\section{F. New Knowledge, New Ways to Help Children and Adolescents}

New developments in pediatric psychopharmacology give us new opportunities to help children and adolescents. Child psychiatrists and other clinicians are learning more from organized clinical trials and from clinical experience.

\section{G. Promoting Access}

Children and adolescents are referred for pharmacological treatment from many places. They come from schools and pediatricians' offices; special education; child welfare and child protection (including DSS); court, probation, or the Department of Youth Services (DYS) (where 50 percent of youths have been exposed to abuse or neglect); and mental health settings. Referral is more likely when symptoms persist or are thought unlikely to respond to psychosocial interventions alone.

But most children with serious emotional disorders are not getting the help they need. Youth from ethnic and racial minorities encounter barriers in seeking and receiving good treatment, as the Surgeon General has reported. Barriers include stigma (that is, prejudice against those with mental illness), mistrust, cost, clinical bias, and cultural suspicion of people with mental illness.

\footnotetext{
1 The US Health Insurance Portability and Accountability Act (HIPAA) defines the person who is legally authorized to make health care decisions as a "Personal Representative." The Personal Representative may be the minor's parent, a court-appointed guardian (with scope to cover health care decisions) or an entity (e.g. DSS or DYS).
} 
We hope information in this booklet will promote access to care for children who are challenged by emotional and mental troubles.

\section{H. This Booklet}

- acknowledges that the field of pharmacotherapy for children and adolescents is growing rapidly;

- provides general information about psychoactive medication for children and adolescents;

- offers principles of good care; and

- describes areas of ongoing controversy.

This document does not indicate which medication should be used for a given kind of mental trouble; that information is provided in available resources like Connor and Meltzer (see references, at VI below). Nor does it recommend particular medications or doses.

\section{CHI LDHOOD AND ADOLESCENT MENTAL HEALTH - AN OVERVI EW}

\section{A. How children grow: Differences in development}

Children and adolescents vary in many ways as they grow up. Some are taller, some shorter. Some eat voraciously and with pleasure; others just eat. Some read early on their own, others later and with effort. For some, puberty comes early, for others, later.

Though everything changes with time, some children "take to" a developmental stage early or easily, others later or with difficulty.

Similarly, differences also occur in behavioral and emotional development. For instance, some children are calmer, some more restless. Some are more clingy, others more independent. For some, mood varies little; others have periods when they feel down or frankly depressed. Some are barely ruffled by upsets and challenges; others "lose their temper," as we say, sometimes for extended times.

Diagnostic statements about children are always made against the background of normal development and the spectrum of abnormal development.

In addition, some diagnoses refer to conditions that are long-lasting (chronic conditions) and those that are short-lived or acute. 


\section{B. When do "differences" amount to "disorders?"}

Children at the extreme of any continuum are said to have "conditions" or "disorders." Doctors and other students of child development have to define how many symptoms it takes to "make" a "condition" or "disorder."

Psychiatrists have written a manual, the American Psychiatric Association's Diagnostic and Statistical Manual, $4^{\text {th }}$ edition (DSM-IV), with the criteria for all of the so-called "mental disorders." These diagnoses are used for classifying the mental disorders that adults, children, and adolescents have. Most of the "disorders" fall in categories (Anxiety Disorders, Mood Disorders, Psychotic Disorders, etc.) derived from adult psychiatry; these disorders do not always fit neatly with the troubles children have. Some of the "disorders" (like AttentionDeficit/ Hyperactivity Disorder, Tic Disorders, Conduct Disorders, Developmental Disorders, and Learning Disorders) usually arise in childhood or adolescence.

Talking about "disorders" may sound odd to parents, teachers, and others who are accustomed to describe children's troubles in terms of differences in development.

Children who do not have all the features of a disorder, as defined by the DSM-IV, are called "subthreshold." They may still need help, even if they do not meet "all the criteria." And defining any child's trouble usually requires more than a DSM diagnosis (see below, III-A).

Children and adolescents with an emotional and behavioral trouble need a careful assessment. Appropriate help usually includes several kinds of intervention, not just medication. Such help is called multimodal treatment.

\section{What can medication help with?}

Medication may help with many problems, but not with everything. Medication may help with disturbances of mood (depressed, elevated mood [hypomania or mania] or unstable mood), disturbances of attention (attention deficithyperactivity disorder), anxiety symptoms including compulsions and flashbacks and other consequences of trauma, some impulse control problems, and confused thinking and perceptions.

Medication will not "bring up to age" a child or adolescent with delayed development. Medication might, however, help with specified symptoms. Medications will not take away the disturbed language and ways of relating seen in children and adolescents with autism or other pervasive developmental disorders. But medication might help with associated problems like aggression, 
obsessive-compulsive behavior or scattered attention. Medication will not help a child or adolescent who has a problem telling the truth. But if lying is a symptom of a major depression, antidepressant medication might help with the depression. Medication will not help with "normal" childhood sadness but may help with clinical depression.

Are we treating "symptoms" (specific problem behaviors) or "disorders" (diagnoses)? This question can be puzzling; the difference between symptoms and disorders can be confusing. Parents bring their children and adolescents for help with symptoms, such as poor school performance, irritability, tics, and depressed mood. But the same symptoms may come from many different disorders, each pointing to differences in brain chemistry and requiring different medication. Because choice of medicine should be guided by a diagnosis, doctors usually diagnose a disorder before recommending a trial of medication.

For instance, a child or adolescent's poor attention may be associated with attention deficit-hyperactivity disorder (ADHD), major depression, psychosis, metabolic disorder, or traumatic brain injury. The indicated medicine will be different with each disorder. I rritability may occur in children and adolescents with brain injury or metabolic disorder, as well as ADHD, depression, Tourette Syndrome, or an adjustment disorder.

But while a specific diagnosis may point to disorder-specific treatment, it is not always possible to base clinical practice on diagnosis alone. Psychiatric diagnoses are "conventions," that is, agreements among physicians about ways to classify people's troubles. These conventions, though shared by doctors and endorsed by insurance companies, differ from the ways that families, and whole cultures, talk about their children's behavior. Professionals must respect a family's ways of telling the story of a child's trouble, in addition to using the "scientific" tools of diagnoses from the Diagnostic and Statistical Manual of the American Psychiatric Association (the DSM).

In addition, DSM diagnoses "fit the patient" less well in child psychiatry than with adults; to adequately describe their adaptive troubles, many children and adolescents are given multiple DSM diagnoses. Future research may refine our present diagnoses, add new ones, or encourage use of different approaches to assessment of children and adolescents (e.g., dimensional diagnosis). In the meantime, clinicians are trying to help children and adolescents with specific symptoms, the targets of prescribed medications.

Parents, guardians and professionals must keep in mind both symptoms and diagnoses and the languages that parents, children and adolescents use to talk about their troubles. 


\section{PRI NCI PLES OF PRACTI CE}

\section{A. Assessment}

\section{A comprehensive assessment: What does it mean?}

Any pharmacotherapy should be preceded by assessment that is individualized, careful, and as comprehensive as necessary.

Assessment of children and adolescents is complicated by:

- the variety of symptoms in childhood. Categorical diagnoses (like those in DSM-IV) may not fully capture the variety of symptoms in children and adolescents. As a result, children and adolescents often receive multiple diagnoses. Even so, families may still feel that the doctor's "language" doesn't fit their own ways of thinking and talking about the troubles of their child or adolescent.

- the way symptoms in children and adolescents change over time. In some conditions, symptoms remain stable for years. In other conditions, symptoms continually change. In still others, there are periods of good functioning interrupted by intervals of symptoms, even serious symptoms.

Medication treatment must be part of a comprehensive plan based on a comprehensive assessment which must include the child or adolescent, the family, and relevant aspects of their world. Data should be gathered in interviews with the child or adolescent and parents (or, absent parents, other caretakers) and from collateral sources (e.g., teachers, protective service workers). In many situations, collateral data (from teachers or protective service workers) are essential. Previous medical records should be reviewed. Discussion with the child's or adolescent's primary care physician is indicated.

Assessment of the child or adolescent should identify:

- presenting symptoms, including the child's or adolescent's own words for symptoms;

- current level of functioning;

- developmental history;

- medical history, including history of substance use, previous treatment history, current psychological examination; and

- any history of neglect, abuse, molestation, or other trauma.

\section{Family, Peers and School, Pediatric Liaison, Psychological Testing}

Assessment of the family should include: 
- family history of mental illness;

- assessment of current functioning and stressors;

- assessment of attitudes toward the child or adolescent's symptoms and needs, including the family's or their culture's ways of describing the child or adolescent; and

- any current or past protective needs.

Assessment of the child's world should describe how the child or adolescent functions with peers and at school. Assessment should identify, for child or adolescent and family, strengths as well as weaknesses. Assessment of children and adolescents should include description of their attitudes to their symptoms and readiness to participate in treatment.

Medical assessment of the child or adolescent should assess:

- general health and development,

- well-child care, and

- possible contributing medical factors.

Medical assessment should include a physical examination.

A call to the primary care physician will provide much of this information and establish connections between physicians.

A more detailed assessment should be offered to children and adolescents with severe symptoms, chronic symptoms, or potential for harm to self or others, and to those for whom ordinary treatment-including treatment with several medications or repeated hospitalizations-have not brought relief. More intensive assessment includes more extensive history from the child or adolescent, family members, and other treating professionals current or past. More intensive assessment may include consultation from specialists in child psychiatry, neurology, neuropsychology, pediatrics, child development, child abuse and neglect, and treatment systems.

Psychological testing should be considered for some children and adolescents to further clarify the child's or adolescent's thoughts and feelings, intellectual capacity, and possible learning problems.

\section{I nterviews, Rating Scales: Using both}

Assessment relies on clinical interviews. In addition, structured methods (like rating scales, questionnaires, and tests) may be used. Commonly used rating scales include the Child Behavior Check List ( $\mathrm{CBCL})$, also known as the Achenbach 
Rating Scale, and the Beck Depression Inventory (BDI), or its pediatric version, the Child Depression Inventory (CDI). All data from rating scales must be interpreted by a clinician. All assessments must recognize the culture of the child or adolescent and family. Assessment and treatment must be appropriately documented and monitored.

\section{Providing more than a DSM diagnosis}

Assessment should provide more than a categorical (DSM) diagnosis. Those assessing children and adolescents should:

- consider the adaptive perspective, meaning that the child's or adolescent's symptoms represent his or her best efforts to cope;

- use a developmental perspective, locating symptoms in the context of longerterm development and adaptation;

- distinguish between transient and longer-term conditions;

- balance accurate description of problems with the identification of strengths and the fostering of hope; and

- balance early detection to facilitate intervention with the risk of inaccurate diagnosis and adverse effects of labeling.

\section{Cultural assessment}

Clinicians should assess the culture of the child or adolescent relative to their own. Clinicians should acknowledge differences in experience or cultural background and consider what knowledge, skills and attitudes they need in order to provide care. The clinician should understand how values, beliefs, customs, language and communication style influence the perception and expression of mental distress, how they shape help-seeking behavior and how their own backgrounds affect the way they hear and respond to children's troubles. The goal is make a partnership or alliance in order to help the child.

\section{B. Planning and Contracting for Treatment}

Contracting for treatment requires time, attention and discussion.

Assessment precedes treatment planning. In assessment process, the clinician specifies target symptoms and goals, selects a medication for initial use and schedules visits for evaluating progress. The clinician then recommends treatment, including medication, to the child or adolescent, parent or guardian. Informed consent can then be sought.

\section{Defining Problems, Target Symptoms, and Goals}


Assessment identifies relevant diagnoses and treatable symptoms. Diagnoses may help clarify the nature of the child's or adolescent's problems and predict their response to treatment.

Treatment is directed to target symptoms, such as anxiety, insomnia, poor concentration, or impulsiveness. It is best to avoid using non-descriptive terms like "acting out" or "behavioral issues." The target symptoms selected should reflect the priorities of the child or adolescent, his or her family, and others (such as teachers or residential staff), in terms of what symptoms are most in need of improvement. Target symptoms are not diagnoses; a given symptom might be seen in a variety of disorders. Indeed, a single diagnosis or disorder may contribute to a number of different symptoms. A diagnosis is one context for a symptom, to be taken into consideration along with other contexts, such as presence of developmental disabilities, medical illness, history of abuse, or disruption of care-taking.

Stating target symptoms in child-friendly language helps the clinician develop a treatment alliance with child or adolescent, family, and others. For instance, a child may prefer to say, "I get really down and feel like dying," rather than, "I have depression with suicidal ideation." Each party to the treatment should state what he or she hopes medication will do. Each should be helped to understand what changes may realistically be expected. Defining target symptoms provides a basis for judging the success of the treatment.

Goals such as improved school performance or peer functioning are too broad to be specific targets for pharmacotherapy. But such goals may help to clarify how medication can help-for example, by improving concentration or reducing social anxiety. Distinguishing between improvement (or lack of it) in target symptoms and progress towards overall goals (or lack of it) can help in defining how medication can contribute to overall progress.

Emergent side effects requiring treatment (like acute dystonias, activation on antidepressants (see section V.C.) or Parkinsonian symptoms) should be anticipated in the treatment plan. When they occur, whether anticipated or not, they must be treated promptly.

\section{Selecting Medications}

A medicine will be recommended to the parent or guardian (usually with the assent and participation of the child or adolescent, according to their developmental level) considering:

a) the class of medicines likely to be effective in treating the disorder;

b) the actions and side effects of individual medicines within that group; 
c) the likely effects of the medicine on the target symptoms;

d) likely side effects or potential toxic effects; and

e) any available data regarding family members' responses to this or similar medications.

The recommendation should reflect the doctor's judgment as to the best medication in general and the preferences of child or adolescent and family as to target symptoms and side effects. Physicians should mention medications other than the one recommended and discuss with all parties what alternatives might be considered if the first agent is not effective.

It is best to begin treatment with a single medication. If a single medication proves to be effective, the response can be attributed to that agent and the child or adolescent will have been exposed to less risk of adverse effects. If treatment with a single agent is ineffective, then it is appropriate to try a different single agent or to add an additional medication.

\section{Planning Treatment and Monitoring}

\section{Monitoring}

Having selected a specific medication, the doctor, child or adolescent, family, and others should share their expectations. Questions include the following:

- How soon should the child or adolescent (and other observers) expect to see benefits from taking the medicine?

- What side effects should be expected, and when? What are the most common side effects? Which are potentially the most serious?

- What should the child or adolescent or family do if they notice side effects or other unexpected effects?

- Who should look for indicators of progress or adverse effects and how? Will monitoring include any formal assessment such as checklists or rating scales?

- How will dosage adjustments be made and in response to which indicators of progress or lack of progress?

- Will laboratory or other medical tests be called for? If so, how and when should they be done?

- How frequently should follow-up visits take place? In response to which circumstances should more frequent or urgent visits be scheduled? 
- Which medical problems might affect the use of the medication?

- What symptoms might be expected when the medicine is stopped?

- Are interactions likely between this medication and others, including alcohol or illegal substances, that the child or adolescent may be taking?

- How will compliance with taking the medication be assessed, monitored, and assured?

- Is there a risk of abusing this medication or of developing dependence? How will this risk be managed?

\section{Anticipating problems}

In treatment planning one must anticipate a course of action if the medicine does not seem or no longer seems to be effective. Acknowledging this possibility at the start of treatment helps prevent disillusionment and increases the chances of continuing collaboration. If all have agreed on target symptoms, on how to measure progress, and on the expected time course, then the doctor, the child or adolescent, the family and others will know when and how to consider alternatives. Explicit planning also decreases the risk that an ineffective agent will be continued longer than appropriate because of unreasonable initial optimism.

\section{Seeking consultation}

The use of expert consultation should be considered if treatment is ineffective. Especially when the prescribing clinician is not a specialist in the treatment of the child's problems, it is useful to be prepared early on to seek consultation from someone with more experience. The need for consultation may also arise when the child or adolescent or his or her family comes from a cultural background markedly different from that of the clinician.

\section{Informed Consent}

Obtaining informed consent completes treatment contracting. Consent should reflect the elements outlined above. The record should document who gives consent; what information is provided; and how, when, where and to whom consent is given.

Who

Typically, for children and adolescents under age 18, medical treatment, including psychoactive medication, must be authorized by the parent or guardian. Except as noted below, there is no legal requirement that a minor child or adolescent give consent or "assent" (that is, implicitly consenting by taking the medicine) to 
treatment. Nonetheless, good clinical practice requires that doctor, parent, and others take account of the child's or adolescent's understanding of and attitude toward the treatment and that they gain as much assent as the child or adolescent can give. The appropriate degree of autonomy to afford the minor varies with age, the type and degree of disability, the parent's attitude, and the urgency of the treatment. In some agencies, some minor children and adolescents are explicitly authorized to refuse psychiatric medication. For example, the Department of Youth Services requires both parental consent and child or adolescent assent to medication, as youths in the DYS take their own medicine when given it by a staff person.

An adolescent 18 or older must give consent to his or her own treatment. Certain adolescents below 18 (emancipated minors and so-called mature minors) also may consent to their own treatment. "Emancipated minor" is defined by statute. The designation of "mature" minor is a clinical decision based on two criteria: the ability of the minor to make an informed decision and the potential harm of informing the minor's parents or guardian of the decision. This concept is most often used in sexual or reproductive health care but may also be relevant when a parent and or adolescent disagree on a recommended medication treatment. Court endorsement of clinicians' designation of the minor as emancipated may be sought but is not required.

Some children and adolescents require special consent. DSS caseworkers consent to "routine treatment" for children and adolescents in the custody of DSS, but not those in voluntary DSS care or committed to the department on a "Child in Need of Services," or CHINS, petition. But by DSS regulation, the prescription of antipsychotic medication is considered to be "extraordinary treatment," requiring explicit court authorization (following a "Rogers petition"). Children and adolescents in the joint legal custody of parents who disagree about treatment may require court assistance to establish authority to treat.

What

Informed consent should specify the child's or adolescent's condition and the proposed treatment. A DMH policy (96-3R) provides relevant language: Clinicians must "disclose (to the patient and/or their legal guardian)... the significant medical information (as of 2003, "protected health information")... that is material to an informed decision by the patient as to whether or not to undergo a proposed treatment." The required information includes:

- indications and target symptoms;

- risks of the recommended medication (including significant, common, and possibly serious or life-threatening side effects); 
- reasonably expectable benefits of treatment;

- expectations of the course of illness without treatment;

- reasonable alternatives to treatment;

- expectations about results of interruptions in treatment; and

- expectations concerning the monitoring of treatment.

\section{How}

To consent to medical care, a person (or his or her parent/guardian in the case of a minor) must be competent. Individuals are presumed competent to make informed decisions unless they are "legally" incompetent (as are children and adolescents under 18), or unless a judge has found them to be incompetent, for instance in a guardianship proceeding. The decision for or against treatment must be a voluntary one, made without undue influence from others; it must be based on a rational ability to understand facts as presented. A decision to forego treatment may or may not be in the person's best interests, as seen by an outsider. Although such decisions are sometimes called "irrational," they are valid as long as the person is competent to make the choice. For instance, a refusal of treatment based on religious beliefs may not be rational to another person but may still be competent. The decision must also be adequately informed, as detailed above.

The medical record needs to document the consent process, including who participates, what information has been conveyed, any concerns that may have arisen, and the final giving of consent for the treatment itself. Standardized signature forms may help in documentation, especially if such forms specify the elements noted above. Generic consent forms are neither necessary nor sufficient documentation for the giving of informed consent.

When

Consent for treatment must be obtained before treatment is begun, unless the treatment is considered to be an emergency (see below). If treatment is undertaken as an emergency, consent must be obtained as soon as possible; treatment cannot continue beyond the emergency without consent. New consent must also be obtained whenever a different medication is introduced and whenever there is any material change in the circumstances of treatment. Such changes include the emergence of side effects, the development of a relevant medical problem, or other developments affecting risk and benefits. Consent for treatment is not a one-time event but rather a process of ongoing discussion with a parent or parents, consenting child or adolescent, or other consenter. 


\section{Where}

A parent should ideally give consent in a face-to-face interview with the treating clinician. Such contact will provide the best opportunity for careful consideration of the questions needing attention. It is less desirable, but sometimes necessary, to discuss treatment recommendations and obtain consent by telephone, as when treatment is being provided in a residential setting and when it is difficult for parent and clinician to meet in person.

To Whom

Consent should be given to the clinician prescribing medication. When the prescribing clinician cannot meet personally with the person providing consent, consent may be given by telephone and written note made of it, signed by the clinician.

\section{Integration of Treatment}

Pharmacotherapy should be coordinated with other interventions. All interventions should comprise an integrated treatment plan. Massachusetts $\mathrm{DMH}$ regulations require providers to obtain authorizations to receive and/or release protected health information (except for emergencies). Authorizations must be signed by the person legally authorized to make health care decisions.

Physicians prescribing medications for children and adolescents should speak to the primary care physician. In addition, clinicians providing psychological services, (for instance, those helping the child or adolescent with self-understanding and self-management) should be aware of the psychiatric diagnoses and pharmacotherapy. They should help the child or adolescent talk with the prescribing child psychiatrist. Similarly, the child psychiatrist should be aware of psychological interventions and use other clinicians' data in assessment and treatment planning. Treatment summaries should reflect each clinician's awareness of the rest of the treatment.

When clinicians share information and use the same language, it helps the child or adolescent make sense of his or her strengths and weaknesses and feel like a whole person. All members of the team should use the same model for understanding the child or adolescent and for bringing about therapeutic change. Multiple autonomous practitioners working in isolation are unlikely to achieve an integrated view of the child or adolescent.

Treatment summaries should be written so that they are understandable to parents and guardians and can be shared with other clinicians, including primary care clinicians and educators, as appropriate. 


\section{Communication and Record Keeping}

Treatment needs to be summarized in a concise record that is easily shared from one treatment setting to another, consistent with the requirements of confidentiality. Authorization for the release of protected health information should be obtained routinely as children and adolescents enter services.

A summary of a child's medication treatment must be more than a list of medications. It must state whether the trial of each medicine was adequate, whether each medicine helped with specific symptoms, and whether the impression of effectiveness was clear or, as often happens, ambiguous.

The parent or legal guardian of a child or adolescent, including custodial agency, should keep copies of all assessments and treatment summaries so that each new provider can review the treatment history. Accordingly, the parent or legal guardian should receive all treatment records from providers promptly. Treating clinicians should not prescribe new treatments until they have reviewed summaries of previous assessments and treatment or spoken to prior treaters while awaiting receipt of the records.

\section{E. Continuity of Care}

Although the problems for which a child or adolescent is prescribed medication are not short-term, many children and adolescents are cared for in multiple short-term placements. Frequent movement requires that assessment and treatment information be efficiently transmitted from one setting to another. The possibility that frequent changes of residence and caregivers may make symptoms worse should be considered as a factor contributing to the child's or adolescent's problems. As the child or adolescent recovers, the goal should be to provide continuity of treatment, including medication, and relationships.

Discontinuity of care may be a special problem for children and adolescents entering the custody of the Department of Social Services (DSS) or detained in a Department of Youth Services facility. Clinicians prescribing psychoactive medications for children and adolescents should be familiar with different agencies' regulations regarding the use of antipsychotic medications. Specifically, if a child or adolescent is taking an antipsychotic medication without judicial review at the time he or she comes under DSS custody, clinicians and others should be alert to the risks of abruptly stopping psychoactive medication and appropriately advise those with responsibility for the child's or adolescent's care. The same considerations apply when children and adolescents on psychoactive medication enter a DYS facility. Interruption or discontinuation of that treatment might cause psychological deterioration and might violate the individual's legal right to treatment. 


\section{I . MEDI CATI ONS I N CURRENT USE}

This section lists medications in current use and their indications. A more extensive discussion of the effectiveness and safety of medications commonly prescribed to children and adolescents has been published in the journal Child and Adolescent Psychiatric Clinics of North America (Pappadopulos EA, Tate Guelzow $B$, Wong $C$, Ortega $M$, Jensen PS, 2004). That review is organized according to class of medications; separate articles review stimulants, antidepressants, mood stabilizers, antipsychotics, anti-anxiety medicines, a class of medicines called adrenergics and a drug that blocks the effects of opiates, called naltrexone.

This section lists names only; some important information, like dose ranges, is excluded. Parents and Personal Representatives should be aware of differences in what is called potency, that is, the therapeutic power of a medication per milligram. A few milligrams of one medication may be more "potent" than hundreds of milligrams of another, even within the same class. Needless to say, parents should not change doses on their own, hoping for a different effect, but must work with the prescribing clinician.

\section{A. Stimulants (or Psychostimulants)}

Stimulants have been used for decades to treat children who are hyperactive and inattentive. These medications reduce hyperactivity and inattention and improve behavioral control and cognitive performance. (These effects are similar to those of caffeine, another stimulant.) Research indicates that stimulants are effective long-term. Children and adolescents vary considerably in their responses to stimulants; many children and adolescents experience only partial symptom relief. (Also see atomoxetine (Strattera) below at III.E. and Attention DeficitHyperactivity Disorder in section V. B.)

$\begin{array}{ll}\text { Generic Name } & \text { Brand Name } \\ \text { Methylphenidate......................... } & \text { Ritalin } \\ \text { Methylphenidate skin patch .............. } & \text { Daytrana } \\ \text { Dexmethylphenidate ..................... Focalin } \\ \text { Sustained-release methylphenidate ....... } \\ \text { Concerta } \\ \text { Ritalin SR } \\ \text { Metadate } \\ \text { Sustained-release dexmethylphenidate }\end{array}$


Dextroamphetamine Dexedrine

Dextrostat

Pemoline Cylert

Dextroamphetamine and Amphetamine Adderal

\section{B. Antidepressants}

Antidepressant medications treat depression (and some kinds of anxiety). They have been prescribed to adults for several decades and to children more recently (see below, in Section V. A.). There are several classes of antidepressants, named for their chemical structure or for the way they are thought to work in the brain. The first antidepressants were called "tricyclic antidepressants." Medications introduced more recently are called "selective serotonin reuptake inhibitors (SSRIs)" because they are thought to act principally by changing the way the brain handles serotonin.

\section{Selective Serotonin Reuptake Inhibitors (SSRIs)}

Selective serotonin reuptake inhibitors (SSRIs) have been widely prescribed for depression and anxiety for children and adolescents. Fluoxetine (Prozac and others), especially when combined when cognitive-behavioral therapy, has been shown to be the most effective (Vitiello, et al, 2006). While the possibility of suicidal ideas or impulses, called "suicidality," led the Food and Drug Administration to require a "black box" warning when antidepressants are prescribed for children and adolescents, there is a greater risk of suicide if depression goes untreated. (The latest FDA recommendation, extending this warning to young adults, is at http://www.fda.gov/cder/drug/antidepressants/default.htm.)

Such warnings are a reminder that all psychoactive medications should be prescribed only after a careful assessment, analysis of possible benefit and risk with and without treatment, fully informed consent (see below), and monitoring of the child once treatment has begun.

Generic Name Brand Name

Fluoxetine Prozac, Sarafem

Sertraline Zoloft

Paroxetine Paxil, Pexeva

Fluvoxamine. Luvox

Citalopram Celexa

Escitalopram Lexapro 


\section{Tricyclic Antidepressants (TCAs)}

Tricyclic antidepressants (TCAs), long the most commonly prescribed antidepressants in adults, have repeatedly been shown to be effective in treating depression and anxiety in adults but not in children and adolescents. Some TCAs are effective in treating other symptoms in children and adolescents, including Attention Deficit Hyperactivity Disorder (ADHD) symptoms and bedwetting. Side effects, especially potential cardiac effects and sudden death in a few children and adolescents on desipramine, make TCAs no longer "first-line" treatment for depression.

\begin{tabular}{|c|c|}
\hline Generic Name & Brand Name \\
\hline Nortriptyline ... & . Aventyl, Pamelor \\
\hline Desipramine... & ...Norpramin \\
\hline I mipramine .... & .............. Tofranil \\
\hline Clomipramine. & .......... Anafranil \\
\hline Amitriptyline... & .................. Elavil \\
\hline Protriptyline.... & ..................Vivactil \\
\hline
\end{tabular}

\section{Monoamine Oxidase I nhibitors (MAOIs)}

Among the other antidepressants, a class of drugs called monoamine oxidase inhibitors (MAOIs) which block the action of an enzyme by that name. They are not considered "first-line" medications for children and adolescents. Newer MAOIs do not have the same side effect risks and may be used in the future to treat depression.

Generic Name Brand Name

Tranylcypromine Parnate

Phenelzine. Nardil

\section{Other Antidepressants}

Bupropion (Wellbutrin) is an antidepressant that is effective for symptoms of ADHD.

Research with other antidepressants in children and adolescents has not demonstrated effectiveness. 
Generic Name

Brand Name

Venlafaxine .Effexor

Nefazodone Serzone

Trazodone Desyrel

Bupropion Wellbutrin, Zyban

Mirtazapine Remeron

Duloxetine Cymbalta

\section{Mood Stabilizers}

Mood stabilizers have been shown to be effective in the treatment of bipolar disorder (also called manic-depressive disorder) in adults. Their use in children and adolescents is controversial (see Section VI., Areas of Controversy). Some studies have suggested an association of weight gain and endocrine side effects (polycystic ovary syndrome) with valproate use in women.

Generic Name Brand Name

Lithium ..Eskalith, Lithonate, Lithobid

Carbamazepine Tegretol, Carbitrol

Valproate Depakote

Gabapentin Neurontin

Lamotrigine Lamictal

Topiramate Topamax

Oxcarbazepine Trileptal

Another class of mood stabilizers, omega- 3 fatty acids, is beginning to be used together with other mood stabilizers in adults. They may be used in the future in children and adolescents.

\section{Antipsychotics}


Antipsychotic medications reduce psychotic symptoms in children and adolescents with schizophrenia and other psychotic disorders, reduce verbal and motor tics in children and adolescents with Tourette Syndrome, and reduce manic symptoms in adults and children and adolescents. They may also have a role in reducing certain abnormal behaviors in children and adolescents with autism and mental retardation.

There are two classes of antipsychotic medications. The older medications (chlorpromazine and others) are called "first-generation antipsychotics" (FGAs). The newer medications, like risperidone, are called "second-generation antipsychotics." (They are also called "atypicals," because they came later.)

First-generation antipsychotics (like chlorpromazine) carry significant risk of movement disorders in both the short and long term. Second-generation medications (like risperidone) cause movement disorders but probably with less frequency; of more concern is their association with serious metabolic risks, including serious weight gain, development of diabetes mellitus, and abnormal secretion of a hormone called prolactin. Weight gain is especially pronounced with olanzapine (Zyprexa) and clozapine (Clozaril). The most effective secondgeneration medication, clozapine (Clozaril), also carries a small but definite risk of bone marrow suppression. Patients on clozapine must have a blood test every other week. Quetiapine (Seroquel) can cause cataracts in adults; children and adolescents on this medication should have regular ophthalmologic exams. All antipsychotics can be sedating, sometimes to a degree that interferes with optimal functioning.

\section{First-generation Antipsychotics:}

Generic Name Brand Name

Chlorpromazine Thorazine

Haloperidol .Haldol

Perphenazine Trilafon

Fluphenazine Prolixin

Pimozide Orap

Thiothixene Navane

Molindone Moban

Loxapine Loxitane

Droperidol Inapsine

Thioridazine ... Mellaril 
Second-generation Antipsychotics (also known as "atypical" Antipsychotics):

Generic Name Brand Name

Risperidone Risperdal

Olanzapine Zyprexa

Clozapine Clozaril

Quetiapine Seroquel

Ziprasidone Geodon

Aripiprazole. Abilify

Paliperidone Invega

In Massachusetts, the prescription of antipsychotic medication is considered "extraordinary treatment." Parents can authorize such treatments, but the Department of Social Services does not authorize such treatment for children and adolescents in its custody. For such children and adolescents, a court order (in response to a Rogers petition) is required (see "Informed Consent," in section IV.B.4).

\section{E. Other Medications}

A few other medications are currently used in children and adolescents, primarily for the treatment of anxiety and/or impulse control problems. They include the medications listed below.

\section{Benzodiazepines}

Benzodiazepines are used to treat anxiety symptoms and sleep problems in adults; research has provided some support for their use in children and adolescents. People taking these medicines long-term may develop problems with tolerance, dependence, and habituation. These medicines are also subject to non-medical use, which can cause undue sleepiness or intoxication, especially when combined with alcohol. Some people taking benzodiazepines develop disinhibited behavior or hallucinations. Withdrawal from benzodiazepines can be difficult.

Generic Name

Clonazepam

Lorazepam
Brand Name

Klonopin 
Alprazolam

Xanax

\section{Others}

Buspirone.......................................... Buspar

Clonidine ....................................... Catapres

Guanfacine......................................... Tenex

Naltrexone ......................................... Revia

Atomoxetine ............................................Strattera

Propanolol...................................................... Inderal

Buspirone can be effective in treating anxiety in adults, with relatively few side effects. There are no controlled studies with children and adolescents. Clonidine has long been used to treat high blood pressure in adults. It has been used extensively with children and adolescents for treating tics, ADHD, aggressive behavior, and sleep problems. Research support for its use in these disorders is mixed. Guanfacine (Tenex) is a newer medication, similar to clonidine but longeracting, less sedating, and possibly more effective in improving attention. It is used in treating impulsiveness and hyperactivity associated with ADHD and other disorders. Research has not demonstrated its effectiveness.

Atomoxetine (Strattera) is a non-stimulant medication for treatment of ADHD.

Naltrexone (Revia), an opiate antagonist, is used to treat self-injurious behavior. Beta-blockers like propanolol (Inderal) are used to treat anxiety, especially performance anxiety, and aggressive behavior.

\section{AREAS OF CONTROVERSY}

In some areas, child psychiatrists are still working to achieve consensus. In other areas, public opinion is divided. Parents, guardians, and children and adolescents should be aware that different practitioners may approach the same problem differently.

\section{A. Use of Antidepressants in Children and Adolescents}

The treatment of depression in children and adolescents is changing. Depression is a serious problem in children and adolescents, as emphasized in the Surgeon General's report. Depression causes suffering, prevents children from participating in school, and puts children at risk of suicide. More depressed 
children are getting help, as parents and professionals have recognized that young people get depression and, when depressed, can benefit from treatment. However, most depressed children and adolescents, like most adults with depression, still do not receive help. Help begins with adults letting a child know they recognize his or her distress and that they do not blame him or her.

What help to offer is increasingly clear. Evidence now indicates that antidepressant medication, the so-called SSRIs, can help depressed adolescents, especially when combined with a psychological treatment, cognitive-behavior therapy. While a few depressed children develop suicidal ideas or impulses while taking SSRIs (mentioned in the "black box warning" from the FDA), it seems that suicides may have increased after the FDA posted a warning and SSRI use decreased. Others point out the great variability in responses to these medicines; even if the many do not respond, many individuals may benefit.

The use of antidepressants in children and adolescents, even more than with other medications, requires careful weighing of potential risks and benefits with and without treatment. Prescription of antidepressants requires careful assessment of these risks and informed decision-making.

The Department of Mental Health urges families and caregivers with young people taking or considering taking antidepressant medication to keep in mind the following good practices:

- Monitoring of the patient by physicians, families and caregivers

- Not abruptly stopping medications without medical advice

- Positive and ongoing communication with the prescribing physician

- Careful risk/benefit analysis when considering antidepressants for young people, including providing information and allowing time for questions and answers from the patient, family and caregivers

- Review of the patient information sheet ("Medication Guide") from the FDA

Also see discussion of "antidepressant activation" in Section C, below.

\section{B. Attention Deficit-Hyperactivity Disorder}

Attention Deficit-Hyperactivity Disorder, although one of the most studied disorders in child psychiatry, is often misunderstood. Typical ADHD symptoms in school-age children and adolescents (restlessness, distractibility, poor attention) resemble the normal behavior of younger children. Controversy exists as to the diagnosis of ADHD and the use of stimulants in children under five years of age. Moreover, considerable misleading and inaccurate information about ADHD and stimulant therapy has been distributed, some of it coming from groups opposed to all treatments in psychiatry. Nonetheless, ADHD is different from normal 
behavior. Children and adolescents with ADHD are at increased risk for academic, social, and legal problems. A consensus statement developed by the National Institutes of Health concluded that ADHD is a valid disorder with considerable costs to individuals and society. Effective treatments exist for ADHD, stimulants being more effective than psychosocial treatments. There is no known prevention for ADHD.

Stimulant medication helps children and adolescents with ADHD to concentrate and focus better. Stimulant medication is as good as or better than behavioral treatment alone. Options in treating ADHD have increased with the demonstration that clonidine (Catapres) and guanfacine (Tenex), alpha-adrenergic agonists, in addition to the stimulants, can be effective, especially for hyperactivity, and with the introduction of a non-stimulant medication (atomoxetine (Strattera)).

Despite this evidence, many children and adolescents with ADHD get no treatment. The Surgeon General (1999) reports that, while ADHD occurs in three to five percent of school-age children, only two to three percent of school-aged children are being treated for ADHD.

\section{MEDICATIONS USED IN ATTENTION DEFICIT-HYPERACTIVITY DISORDER}

\section{$\underline{\text { STIMULANTS }}$}

Short-acting:

Methylphenidate (Ritalin)

Desxmethylphenidate (Focalin)

Dextroamphetamine (Dexedrine)

Pemoline (Cylert)

Intermediate-acting:

Sustained-Release Methylphenidate (Metadate ER)

Methylin ER

Ritalin SR

FocalinXR

Daytrana patch

Long-acting:

Concerta

Sustained-Release Methylphenidate (Metadate CD)

Dexedrine Spansules

Amphetamine Mixture (Adderall XR) 


\section{ALPHA-ADRENERGIC MEDICATIONS}

Clonidine (Catapres)

Guanfacine (Tenex)

\section{ANTIDEPRESSANTS}

SSRIs, TCAs, Bupropion (Wellbutrin)

\section{$\underline{\text { OTHER }}$}

\section{Atomoxetine (Strattera)}

Several points bear emphasis. First, children and adolescents with ADHD tend to have other problems as well. Accordingly, those treating children and adolescents with ADHD may make several diagnoses and recommend more than one medicine. Second, several factors other than ADHD may make it hard to concentrate. The child or adolescent who is hungry, or who is worried about his or her safety, or who has flashbacks or thought disorganization, or whose school program does not match his or her needs, will have difficult attending to schoolwork. Amid such possibilities, the possibility of ADHD must be considered in the context of an overall assessment of the child and his or her life situation. Third, the benefit of stimulant medication may only become apparent when external and internal turmoil has subsided. That is, a child or adolescent may benefit from stimulants, but not while being abused or neglected or while struggling to learn in when no one knows he has a learning disability, or while in an acute psychotic state. The child or adolescent who does not appear to benefit from stimulants at such a time may, when the other problems improve, benefit. Medication treatment for ADHD should not be undertaken as if the other problems did not exist. Fourth, in a child or adolescent receiving multiple medications, the interaction between the medications must be carefully monitored.

With ADHD as with other disorders, different clinicians, even with the same data, may make different treatment recommendations.

\section{Bipolar Disorder}

Recent research indicates that bipolar disorder occurs in children and adolescents. Its features include emotional lability, especially tantruming, as well as hypersexuality and suicidal behavior. Treatment with mood stabilizers may be helpful. Clinicians disagree about the criteria for this diagnosis in children and adolescents and whether to recommend treatment with mood stabilizers. A family history of bipolar disorder in a child or adolescent presenting with serious 
problems with mood or temper regulation warrants close monitoring of any antidepressant use and a possible trial of a mood stabilizer. Inappropriate diagnosis of bipolar disorder, on the other hand, may expose the child or adolescent to unwarranted medication and undercut the child or adolescent's efforts at self-understanding and self-management. When a child or adolescent, or the family, say, "I'm (he's) bipolar-" they may stop thinking about the course, meaning, and management of mood changes or emotional dysregulation.

Related to bipolar symptoms is so-called "antidepressant activation." This term is used when the child or adolescent taking an antidepressant develops increased activity, irritability, insomnia, hypersexuality, grandiosity, hallucinations or rapid talking. Such activation does not make the diagnosis of bipolar disorder nor predict its emergence later; it may just be a medication side effect unrelated to bipolar disorder. Some children and adolescents may benefit from antidepressant medication but only when also taking a mood stabilizer. For such children and adolescents, a mood stabilizer may be prescribed along with an antidepressant.

\section{Use of Antipsychotics (also known as Neuroleptics)}

Some clinicians prescribe antipsychotic medications only for frank psychotic thought disorder. Others argue for a broader range of use in children and adolescents, helping those with severe, confusion or uncontrollable emotional flooding. Both first- and second-generation antipsychotics have serious effects. Their prescription is not to be undertaken lightly.

Antipsychotic medications should not be prescribed for children and adolescents as a substitute for acknowledging and treating their other challenges. Many challenges-such as unrecognized abuse or molestation, post-traumatic states, severe caretaking deficits, unrecognized visual or hearing impairment or other learning disability, or unacknowledged family conflict-can make children and adolescents feel overwhelmed and disorganized. Too hasty assessment of such children and adolescents can result in injudicious prescription of possibly unwarranted medication, as well as delay in recognizing and addressing other adversities in the life of the child or adolescent.

On the other hand, when the efforts of the child or adolescent to cope with adversity are compounded by inner disorganization, environmental interventions may still leave them too disorganized to take advantage of the opportunities available. A trial of antipsychotic medication may dramatically enhance such individuals' ability to cope.

A comprehensive plan must ascertain that antipsychotic medication, if offered, has indeed made a difference in the child's or adolescent's coping and that such use is continued no longer than necessary. 


\section{E. Post-Traumatic States}

While we know much about post-traumatic symptoms in children and adolescents, researchers are still evaluating what are the best treatments for traumatized children and adolescents. It is important to differentiate between "simple PTSD" which follows a discrete traumatic event, and "complex PTSD," which follows multiple traumas, over time, often not acknowledged, and associated with a wide variety of symptoms. Treatment options include psychotherapy, especially cognitive-behavior therapies (CBT), pharmacotherapy and the newer physical therapies such as eye-movement desensitization and reprocessing (EMDR). Medications like clonidine and the SSRIs (fluoxetine, etc) help children and adolescents cope with the symptoms that come from trauma. The use of such medications in children and adolescents rests on clinical experience, not on controlled clinical research trials.

Abused and neglected children and adolescents often present with extreme fragility, withdrawal, aggression and mood instability that do not meet full criteria for PTSD but that require intervention. Treatment for such children and adolescents should include protective intervention, protective acknowledgement, and psychological support by experienced therapists. Interventions should not be limited to the use of medications, nor should diagnoses with lasting significance be made while the trauma-surviving child is just emerging from an unprotective traumatic environment.

Symptoms such as "hearing voices" are often present in traumatized children and adolescents. Such hallucinosis is not in itself an indicator of psychotic illness and may not require anti-psychotic medications, with the attendant risks. Children and adolescents with a history of trauma may also respond to subtle reminders of the trauma with behavioral outbursts and emotional instability resembling that seen in affective disorders. Mood stabilizers, which are accompanied by fewer risks than antipsychotics, may be helpful in treating such children and adolescents.

A thorough assessment of the child or adolescent, noting current and past trauma, is necessary to identify factors that may respond to medications, as well as those that require psychotherapy and environmental interventions.

\section{F. Children and Adolescents with Developmental Disabilities}

Psychiatric symptoms in children and adolescents with developmental disabilities present many clinical challenges, including whether to use medication. ${ }^{2}$ It is

\footnotetext{
${ }^{2}$ Identifying sources of help for children with developmental disorders, including autism and Asperger's syndrome, can be confusing. Federal regulations locate responsibility for such children, including their mental health problems, in the public schools (the so-called Local Education
} 
unclear which diagnostic criteria should be used in this population, especially in children and adolescents with IQ's below 50. Diagnostic uncertainty notwithstanding, children and adolescents with developmental disabilities are more likely to have multiple disorders.

Developmentally delayed children and adolescents with psychiatric symptoms should have a thorough assessment in a multidisciplinary setting and should be offered comprehensive therapy with emphasis on specialized behavioral interventions.

We do not know enough about the safety and effectiveness of psychoactive medications in children and adolescents with developmental disabilities; there are only a small number of sound studies. Many medications have been recommended; few have been shown to be effective in controlled trials. Nevertheless, depending on the circumstances of the individual child or adolescent (and possible associated psychiatric comorbidity), antipsychotics or mood stabilizers may be recommended. A recent well-designed study demonstrated a positive response to risperidone (Risperdal) in children with developmental disorders.

Pharmacological intervention is often recommended when aggressive or selfinjuring behavior is so intense, frequent or persistent as to threaten the child or adolescent or those nearby. It may also be recommended when behavior is so disruptive that the child or adolescent cannot use school or occupational, physical, speech, or interactional therapies. Except in emergencies (see below, Section G), clinicians should not prescribe something immediately "to take the edge off" the challenging behaviors in lieu of a diagnostic assessment that will complete the overall picture of the child or adolescent, including delineation of the factors that contribute to the behavior. Parents, teachers, and other caregivers may need considerable support during this phase, in order to ensure their cooperation with the recommended treatment plan.

It is advisable to treat each case on an individual basis, "as a single case study, starting with the safest and most commonly used medications in low doses and increasing the dose gradually" (Santosh \& Baird, 1999). In deciding to continue treatment or revise the plan, adults should use reports of behavioral changes, including the child's own assessment, the child's tolerance for side effects, and any idiosyncratic reactions to the medication.

Authority). In Massachusetts, developmentally disabled people over age 22 are served by the Department of Mental Retardation. In this booklet, we address the principles that guide their medication treatment, not where services are to be found. 


\section{G. Extreme Behavioral Loss of Control (Aggressive and Self-I njurious)}

As mentioned above, medication treatment in children and adolescents is based more on clinical experience than on controlled clinical trials-especially when dealing with children and adolescents with aggressive or self-harming behavior. For many such children and adolescents, the need for urgent intervention is often clearer than the diagnosis. With or without diagnostic clarity, medication is often an important part of their treatment. Such treatment must, like any treatment, be part of a carefully developed plan based on comprehensive assessment. When the diagnosis is uncertain, specifying the factors that contribute to the problem may help to summarize a formulation and guide treatment. Target symptoms should be identified, as in any other treatment, and medications judiciously introduced, with the results monitored according to an agreed-upon plan.

It is essential to examine the context in which aggressive or self-harm behavior is occurring. Medication should never be prescribed for a child or adolescent with extreme behavioral loss of control without an inquiry into context and interactions. Assessment should also be alert to the possibility of undiagnosed medical disorder (endocrine-metabolic or toxic), of substance use including caffeine or withdrawal from nicotine, and of unrecognized psychiatric disorder, such as post-traumatic or other anxiety disorder, depression or bipolar disorder, or unrecognized psychosis.

In prescribing any agent for extreme behavioral loss of control, the clinician should balance the urgency of the situation against the desirability of acquiring more understanding before taking action. The clinician should also consider whether the treatment will constitute an emergency, and, if so, whether treatment will be voluntary or involuntary (see Section $\mathrm{H}$, below).

In general, pharmacological treatment of extreme behavioral loss of control starts with less toxic, shorter-acting medications: sedatives (like diphenhydramine [Benadryl and others]), alpha-agonists and beta-blockers. Longer-acting medications and those with more side effects (mood stabilizers, antidepressants, naltrexone [Revia], antipsychotics) are recommended either when a specific disorder is diagnosed (e.g., depression, bipolar disorder, or psychosis) or as an empirical trial when shorter-acting or less toxic medications have failed or are deemed unlikely to be effective.

\section{H. Involuntary Treatment}

This guideline may give the impression that medication treatment is always voluntary, but involuntary treatment also occurs. 
A competent child or adolescent or parent/guardian has the right to refuse treatment. Therefore, involuntary treatment may be given only when a court has found the parent/guardian to be incompetent to provide informed consent or that the parent/guardian is endangering the child or adolescent, as in a life-threatening situation.

When a parent/guardian gives informed consent for treatment and the minor child refuses treatment, it is incumbent upon the treating clinician to attempt to gain the minor's assent (although there is no legal obligation to do so). If a parent or legal guardian refuses to consent to treatment and a clinician judges that this refusal places the well-being of the child or adolescent in jeopardy, the clinician should consider seeking assistance from the Department of Social Services or asking the court to appoint a new guardian.

Emergency involuntary medication treatment may be given in two contexts. In one setting, chemical restraint medication is given involuntarily to assist in containing a child with immediately threatening behavior. At other times, emergency involuntary medication may be given to prevent immediate, substantial and irreversible deterioration of serious mental illness.

Chemical restraint, like all forms of restraint, may only be used in emergencies, when extreme violence, personal injury, or attempted suicide has occurred or been threatened. Such measures can be used only when there is a substantial risk of serious self-destructive behavior or of serious physical assault or when a serious physical assault has occurred. A child or adolescent may be given chemical restraint only on the order of an authorized physician. This physician must have determined that such chemical restraint is the least restrictive, most appropriate alternative available. The physician can make this determination while present in person or after telephone consultation with a physician, registered nurse or certified physician assistant who was present at the time of the emergency and who personally examined the child or adolescent (See DMH Regulations 104 CMR 27.12). [In schools, the Department of Education regulates the use of restraint and requires authorization by a physician and parent.]

Apart from situations requiring use of restraint, emergency treatment occurs very infrequently. It may occur only when the physician believes that delaying treatment in order to obtain a court order for the treatment poses the risk of the substantial and irreversible deterioration of the child or adolescent's serious mental illness. There are very few instances in which this threshold is met.

\section{Adverse Side Effects}

All prescribing child psychiatrists and other clinicians are concerned about possible adverse side effects of medication. Opinions differ as to how to weigh the 
advantages and disadvantages of a course of treatment in a given child or adolescent. The final decision, of course, rests with the parent or guardian, with the advice of clinicians. Once treatment is underway, vigilance is indicated for emergent side effects, including unusual or idiosyncratic reactions. For instance, mood stabilizers/anticonvulsants like valproate (Depakote and others), which help most children to manage emotional lability, may cause increased irritability and temper tantrums in some.

Of particular concern are the metabolic side effects of second-generation antipsychotics (see above, II. D.). The American Psychiatric Association and American Diabetes Association (2004) have made recommendations for the use and monitoring of these medications.

\section{CLIENT/ PARENT EDUCATION}

Parents, full of questions as they seek the best care for their children, confront an unprecedented volume of information, including on the internet, of varying quality. There is no substitute, in this flood of information, for personal relationships with trusted providers (primary care physicians, social workers, or child psychiatrists). In addition to those personal resources, the following books, articles, and websites are recommended:

Connor DF, Meltzer BM. Pediatric Psychopharmacology: Fast Facts. New York: WW Norton, 2006.

Dulcan MK ed. Helping Parents, Youth, and Teachers Understand Medications for Behavioral and Emotional Problems. A Resource Book of Medication Information Handouts. Washington DC: American Psychiatric Press, 1999.

Findling RL, Steiner $\mathrm{H}$, Weller EB. Use of antipsychotics in children and adolescents. J Clin Psychiatry 2005; 66 Suppl 7:29-40. Review.

Greene RW. The Explosive Child: A new approach for understanding and parenting easily frustrated, "chronically inflexible" children. New York: Harper Collins, 1998

McClellan J, Kowatch R, Findling RL; AACAP Work Group on Quality Issues. Practice parameter for the assessment and treatment of children and adolescents with bipolar disorder. J Am Acad Child Adolesc Psychiatry 2007; 46:107-125.

National Institute of Mental Health - Health Information http://www.nimh.nih.gov/ 
US Public Health Service. Report of the Surgeon General's Conference on Children's Mental Health: A National Action Agenda. Washington DC: Department of Health and Human Services, 2000. Also available at http://surgeongeneral.gov/cmh/childreport.htm

Vitiello B, Rohde P, Silva S, Wells K, Casat C, Waslick B, Simons A, Reinecke M, Weller E, Kratochvil C, Walkup J, Pathak S, Robins M, March J; TADS Team. Functioning and quality of life in the Treatment for Adolescents with Depression Study (TADS). J Am Acad Child Adolesc Psychiatry 2006; 45:1419-26.

Wilens TE. Straight Talk about Psychiatric Medications for Kids. New York: Guilford Press, 1999.

www.aacap.org - American Academy of Child and Adolescent Psychiatry - Facts for Families

\section{Other References of interest}

American Diabetes Association, American Psychiatric Association, et al. Consensus development conference on antipsychotic drugs and obesity and diabetes. Diabetes Care 2004; 27:596-601.

Levine MD, Carey WB, Crocker AC, eds. Developmental-Behavioral Pediatrics. Philadelphia: Saunders, 1999.

Martin A, Scahill L. (eds.) Psychopharmacology. Child and Adolescent Psychiatric Clinics of North America 2000. Vol 9, No.1.

NIH Consensus Statement, Vol 16, No. 2, Nov 16-18, 1998. Diagnosis and Treatment of Attention Deficit Hyperactivity Disorder (ADHD). Bethesda, MD: National Institutes of Health, 1998.

Pappadopulos EA, Tate Guelzow B, Wong C, Ortega M, Jensen PS. A review of the growing evidence base for pediatric psychopharmacology. Child Adolesc Psychiatr Clin N Am. 2004;13:817-55, vi.

Riddle MA, Kastelic EA, Frosch E. Pediatric psychopharmacology. J Child Psychol Psychiatry 2001:42:73-90.

Roberts RE, Attkisson CC, Rosenblatt A. Prevalence of psychopathology among children and adolescents. American Journal of Psychiatry 1998; 155:715-725.

Santosh PJ, Baird G. Psychopharmacotherapy in children and adults with intellectual disability. The Lancet 1999; 354:233-42. 
US Department of Health and Human Services. Mental Health: A Report of the Surgeon General. Rockville, MD: U.S. Department of Health and Human Services, Substance Abuse and Mental Health Services Administration, Center for Mental Health Services, National Institutes of Health, National Institute of Mental Health, 1999.

Vitiello B, Bhatara VS, Jensen, PS, eds. Current Knowledge and Unmet Needs in Pediatric Psychopharmacology. Special Section. Journal of the American Academy of Child and Adolescent Psychiatry 1999; 38. 501-565.

Zarin D, Suarez AP, Pincus HA, Kupersamin A, Zito J M. Clinical and treatment characteristics of children with attention deficit/hyperactivity disorder in psychiatric practice. J Am Acad Child Adolesc Psychiatry 1998;37:1262-70.

Zito J M, Safer DJ, dos Reis S, Gardner JF, Boles M, Lunch F. Trends in the prescribing of psychotropic medications to preschoolers. JAMA 2000;283:10251030.

\section{I. Members of Working Group (identifications as of 2001)}

John Backman, MD

Child and Adolescent Psychiatrist

Central Area Office

Massachusetts Department of Mental Health

Donald Condie, MD

Child and Adolescent Services

Massachusetts General Hospital

Mary Ellen Foti, MD

Department of Mental Health Deputy Commissioner of Medical Services

Steven Feldman, MD

Massachusetts Behavioral Health Partnership

Gordon Harper, MD (Chairman)

Child and Adolescent Services

Massachusetts Department of Mental Health

Lisa Lambert

Parent Professional Advocacy League 
Isa Woldeguiorguis

Massachusetts Department of Social Services

Bruce Meltzer, MD

UMMC Continuing Care Inpatient Unit

Westborough State Hospital

Resa Brandfonbrener (staff)

Child and Adolescent Services

Massachusetts Department of Mental Health

Former Members (affiliations as of 2001)

Richard Barnum, MD

Boston J uvenile Court Clinic

Shay Beauregard, RN

Health and Education Services

Paul Dagincourt, MD

Westwood Lodge Hospital

Ken Dawson, MD

Harvard Community Health Plan

Cindy Nichols

Parent Professional Advocacy League

Susan Pederzoli

Massachusetts DSS

Tina Render, MD

UMMC Continuing Care Unit

Glenn Saxe, MD

Boston Medical Center

Yvette Yatchmink, MD, PhD

Massachusetts Division of Medical Assistance 